\title{
XVIII. Notes on the electromagnetic theory of light.-I. Geometrical properties of the wave- surface \&c. II. Reflexion and refraction at the boundary of crystals, treated by a theorem of Sir William Rowman Hamilton
}

\author{
Prof. A. McAulay
}

To cite this article: Prof. A. McAulay (1900) XVIII. Notes on the electromagnetic theory of light.-I. Geometrical properties of the wave-surface \&c. II. Reflexion and refraction at the boundary of crystals, treated by a theorem of Sir William Rowman Hamilton, Philosophical Magazine Series 5, 49:297, 228-242, DOI: 10.1080/14786440009463839

To link to this article: http://dx.doi.org/10.1080/14786440009463839

Published online: 21 Apr 2009.

Submit your article to this journal ¿

ЦIl Article views: 3

Q

View related articles $\asymp$ 
were so defined, it would eo ipso facto cease to be " molecular ungeordnet." But the working assumption which he actually makes use of in the special case, as a deduction from hì general assumption, is the same as that used by Dr. Watson and other writers, namely that, regarding $f$ and $\mathrm{F}$ as the chances that a molecule shall have velocities between the limits (1) or (2), these chances are independent of the relative position of the molecules-at least that this is the case for molecules approaching collision. Hence $f$ and $\mathrm{F}$ being independent, the number per unit of volume and time of collisions between members of the class $f$ and members of the class $\mathrm{F}$ is proportional to $F f$. And from this, by a well-known method, it is proved that

$$
\begin{aligned}
& f^{\prime}=c \epsilon^{-h m\left(u^{2}+v^{2}+x^{2}\right)} \\
& \mathbf{F}=\mathrm{C} \epsilon^{-h \mathbf{M}\left(\mathrm{U}^{2}+\mathrm{V}^{2}+\mathrm{W}^{2}\right)}
\end{aligned}
$$

and therefore $m \overline{u^{2}}=\mathrm{M} \overline{\mathrm{U}^{2}} \& \mathrm{ce}$, which is the law.

But the question arises whether the state of things assumed by Boltzmann to exist, from which the theorem of equal partition of energy follows as a mathematical deduction, can exist, and continue to exist, in fact in a finite system of molecules left to their own mutual actions for infinite time undisturbed from without.

I think Lord Rayleigh's argument, p. 109 et seq., requires some assumptions :-

(1) Systems on the same path move independently of one another.

(2) Systems cannot exchange paths; or if they can we must assume a law of interchange.

I think there may be other constants besides E, e. $g$. if two elastic spheres with velocities $u_{1}, u_{2}$, \&c. collide, $u_{1} u_{2}+v_{1} v_{2}+w_{1} u_{2}$ is constant as well as $\mathrm{E}$.

Does not the method prove too much? namely that not merely the law of equal partition (p. 115), but in effect the distribution $\epsilon^{-h \mathbb{R}}$ must hold for all states of matter.

XVIII. Notes on the Electromagnetic Theory of Light.-I. Geometrical Properties of the Wave-Surface \&c. II. Reflexion and Refraction at the Boundary of Crystals, treated by a Theorem of Sir William howan Hamilton. By Prof. A. MoAulay, University of Tasmania *.

1. Geometrical Properties of the Wave-Surface \&s. M R. OLIVER HEAVISIDE (' Electrical Papers,' vol. ii. 1 p. 1 ; Phil. Mag. xix. June 1885, p. 397) first, J believe, investigated the properties of the general electro-

* Communicated by the Author. To be read at the Meeting of the Australasian Association for the Advancement of Science, January 1900. 
magnetic wave-surface. The present writer (Phil, Mag. xlii. Sept. 1896, p. 224) pointed out that it is an orthogonal projection-in two different ways - of the Fresnel surface. I do not know of other writers who have contributed to this particular department; but I suspect that this is due to my jgnorance, and not to the absence of literature.

The following statements are either explicitly made in, or are obviously deducible from, the text of Basset's ' Physical Opties.' In that particular form of Maxwell's ElectroMagnetic Theory of Light in which the media are supposed to be non-magnetic, we have exactly the same analytical conditions, so far as plane waves in a non-absorbent medium are concerned, as in three other theories of light, viz.: (1) Fresnel's (2) MacCullagh's, (3) Lord Kelvin's (contractile æther). To be more precise :-(1) The electromagnetic displacement, $\mathbf{D}$, is the exact mathematical equivalent of Fresnel's displacement; (2) the electromagnetic M.M.F., $\mathbf{H}$ (or, what is the same in the present case, the magnetic induction B), is the exact mathematical equivalent of MacCullagh's displacement; (3) the electromagnetic E.M.F., E, is the exact mathematical equivalent of Lord Kelvin's displacement.

In the second and third cases the mathematical equivalence extends, not only to the properties of plane waves within a medium, but also to the boundary conditions between two media. this is true in the first case also, in so far that the laws of reflexion and refraction at the boundary of isotropic transparent media, which result from the two theories, are the same.

If only for this extraordinary property of being mathematically equivalent to so many distinct and celebrated theories, the electromagnetic theory is worthy of study.

It is to be remarked that it is only a particular form (certainly a very general particular form) of the electromagnetic theory which has these wide and exact mathematical analogies with other theories. Mr. Heaviside has taught us to look, in Electromagnetism, for a symmetrical dual interpretation of results, the electric and magnetic quantities providing the duality. It may be anticipated that in the general electromagnetic theory of light, insight will be obtained by adhering to the symmetrical dual interpretation.

This first part of the present paper is mainly concerned with geometrical properties of the wave-surface exhibiting this symmetry. Incidentally a new presentation of the fundamental analysis will be given.

Four geometrical theorems are enunciated, and immediately after the enunciation of each, some of its obvious geometrical consequences are detailed. The proofs of the propositions are then given. 
Terminalogy and Notation. - Intensity is understood to mean energy per unit volume. $\mathbf{D}, \mathbf{E}, \mathbf{B}, \mathbf{H}$ each $\div$ (intensity) denoted by $\delta, \epsilon, \beta, \gamma$ respectively. If it is thought desirable to give names to these vectors, they may be called the quasiunit displacement, the quasi-unit E.M.F., \&c. The terms $\mathbf{D}$-oid, E-oid, \&c. used in Prop. I. below may be regarded as convenient abbreviations of displacement-ellipsoid, E.M.F.ellipsoid, \&c. The vector of ray-velocity and the index-vector will be denoted by $\rho, \sigma$ respectively; that is to say the magnitude of $\rho$ is the velocity of propagation of a ray parallel to $\rho$, and the magnitude of $\sigma$ is the reciprocal of the corresponding wace-front velocity, $\sigma$ itself being perpendicular to the wavefront and in the direction of its motion. The locus of the extremities of $\rho$ and $\sigma$, supposed drawn from a given origin $O$, are, as usual, called the wave- and index-surfaces respectively. To fix the ideas, we may suppose the unit of length to be $1 \mathrm{~cm}$, and the unit of time to be $1 /\left(3 \times 10^{10}\right)$ of a second. Thus in vacuo both wave- and index-surfaces will be spheres each of about $1 \mathrm{~cm}$. radius.

Prop. I. The loci of the extremities of $\delta, \epsilon, \beta, \gamma$, supposed all drawn from $\mathrm{O}$, are ellipsoids with common centre $\mathrm{O}$. These ellipsoids will be called the D-oid, E-oid, B-oid, and H-oid respectively.

Prop. II. The D-oid and E-ond are reciprocal polars; and $\delta$ and $\epsilon$ are corresponding vectors of them. $[\mathrm{S} \delta \epsilon=-1$, $0=\mathrm{S} \delta d \epsilon=\mathrm{S} \epsilon d \delta$.]

The $\mathbf{B}$-oid and $\mathrm{H}$-oid are reciprocal polars; and $\beta$ and $\gamma$ are corresponding vectors of them. $[\mathrm{S} \beta \gamma=-1,0=\mathrm{S} \beta d \gamma=\mathrm{S} \gamma d \beta$.]

The wave-surface and index-surface are reciprocal polars; and $\rho$ and $\sigma$ are corresponding vectors of them. $[\mathrm{S} \rho \sigma=-1$, $0=\$ \rho d \sigma=\mathbf{S} \sigma d \rho$.]

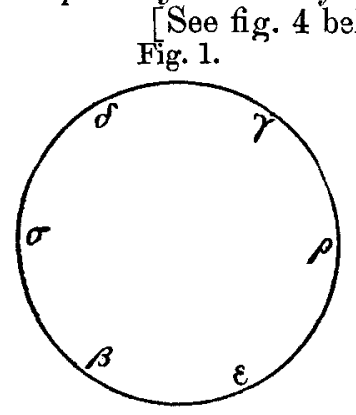

The last of these three statements results of course from the definitions of the vector of ray-relocity and of the indexvector. It is given here merely to bring out the similarities between the six vectors. 
If the symbols $\rho, \gamma, \delta, \sigma, \beta, \epsilon$ be arranged in this cyelic order (counter-clockwise in fig. 1) uniformly round a circle, it will be noticed that the three pairs of corresponding vectors mentioned in Prop. II. are those at extremities of diameters. And further :-

Prop. III. When so arranged, each vector is the vectorproduct of its two neighbours taken in the cyclic order named. That is to say, $\rho=\mathrm{V} \epsilon \gamma, \gamma=\mathrm{V} \rho \dot{\delta}$, \&c.

For some purposes-especially to see easily the simplifications which occur when $B$ is parallel to $\mathbf{H}-$ it is better to

Fig. 2.

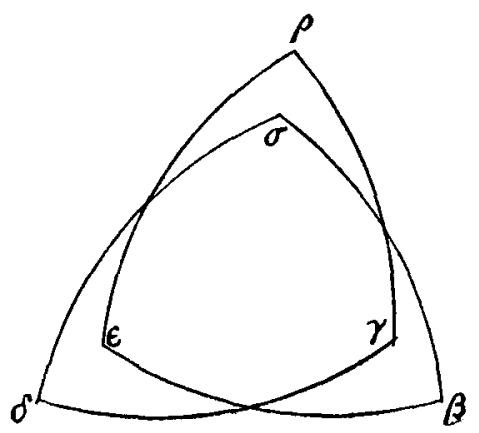

illustrate this by fig. 2. The curved lines of this figure are meant to represent quadrantal ares on a sphere (looked at from the outside), and the points marked $\rho, \gamma, \ldots$ are the points where lines from the centre parallel respectively to the vectors $\rho, \gamma, \ldots$ meet the sphere.

When $\mathbf{B}$ is parallel to $\mathrm{H}$ it will be noticed that fig. 2 becomes fig. 3 .

Fig. 3.

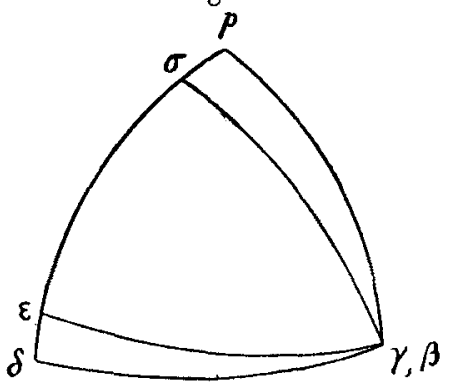

In fig. 4 the plane of the paper is taken as the plane which, in fig. 3 , contains $\rho, \sigma, \epsilon, \delta$. In this case $\beta$ and $\gamma$ must be drawn upwards from $O$. The figure indicates the mutual 
relations of $\rho, \sigma, \delta$, and $\epsilon$ and the corresponding surfaces, as given by Prop. II. An inspection of the figure shows that $\delta$ is parallel to the projection of the ray on the wave-front,

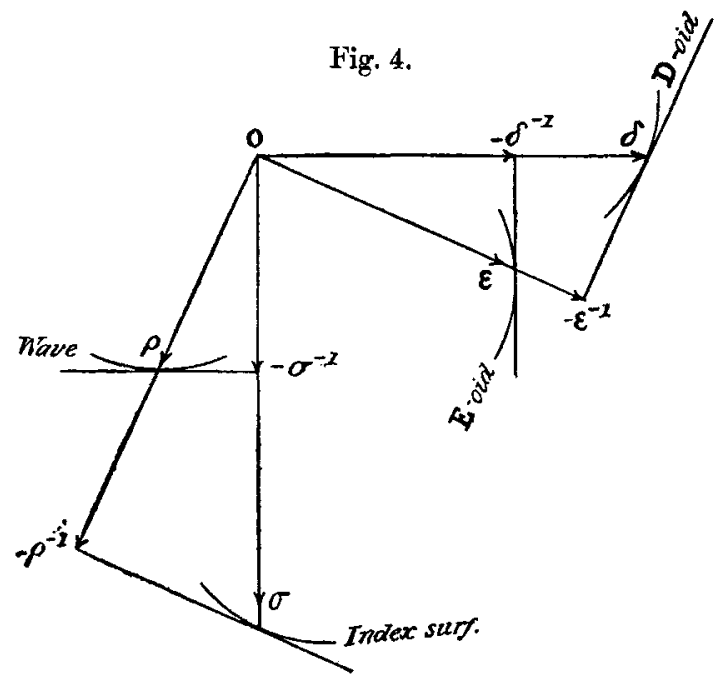

that $\epsilon$ is parallel to the projection of $\sigma$ on the tangent-plane of the index-surface, and that $\mathbf{H}$ and $\mathbf{B}$ are perpendicular to all the other vectors.

By Prop. II. it will be noticed that not more than three of the six surfaces, wave-, D-oid, \&c., are independent. As a matter of fact two, and but two, are. These may be taken as the $\mathbf{E}$-oid and $\mathbf{H}$-oid (or as the $\mathbf{D}$-oid and B-oid); for when these are given, the D-oid and B-oid are given by Prop. II., and the wave-surface and index-surface by Prop. IV.

Prop. IV. $\epsilon$ and $\gamma$ are, in direction, conjugate to one another, with regard to both the $\mathbf{E}$-oid and $\mathbf{H}$-oid.

$\delta$ and $\beta$ are, in direction, conjugate to one mother, with regard to both the D-oid and B-oid.

This proposition (when the E-oid and $\mathbf{H}$-oid are given) suffices to determine all of the four $\epsilon, \gamma, \delta, \beta$ when the direction of any one is given. For the ellipsoids serve by Prop. I. to give the magnitude of each when its direction is known, so that it is only necessary to determine the direction of each. Suppose the direction of $\epsilon$ given : since $\gamma$ is conjugate to $\varepsilon$ with regard to the E-oid, $\gamma$ is confined to one plane; since it is also conjugate with regard to the $\mathbf{H}$-oid, it is confined to another plane. Its direction therefore is determinate. [Its sense may have either of the two values 
corresponding to this direction; but on account of the relation $\rho=\mathrm{V} \epsilon \gamma$, the senses will correspond to propagations of disturbance in opposite directions.] When $\epsilon$ and $\gamma$ are known, $\delta$ and $\beta$ are known by Prop. II.

Also, when $\epsilon$ and $\gamma$ are known, $\rho$ is known; since $\rho=V \epsilon \gamma$, and also $\sigma$, since $\sigma=\mathrm{V} \delta \beta$. Thus when the direction of any one of the four $\epsilon, \gamma, \delta, \beta$ is known, all six vectors are known.

But Prop. IV. also suffices to determine all six when the direction of $\rho$ or of $\sigma$ is known, and gives us geometrical constructions for the wave- and index-surfaces.

Thus if the direction of $\rho$ is given, the plane of $\epsilon$ and $\gamma$ (which is perpendicular to $\rho$ ) is given. This plane cuts the E-oid and H-oid in known ellipses, with regard to both of which $\epsilon$ and $\gamma$ are conjugate. Let ( $\mathrm{fg} .5$ ) $O \mathrm{H}_{1} \mathrm{E}_{2}, \mathrm{OE}_{1} \mathrm{H}_{2}$

Fig. 5.

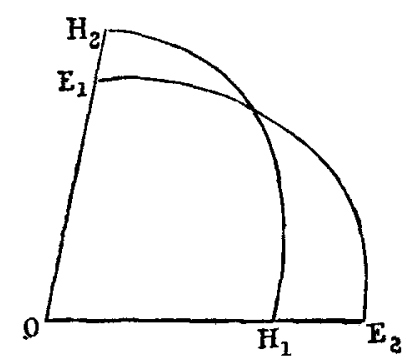

be the common conjugate semi-diameters of these ellipses, $\mathrm{OE}_{1}, \mathrm{OE}_{2}$ belonging to the E-oid, and $\mathrm{OH}_{2}, \mathrm{OH}_{2}$ to the H-oid. $\epsilon$ may be either $\pm \overline{\mathrm{OE}_{1}}$ or $\pm \overline{\mathrm{OE}_{2}}$. If we take $\epsilon=\overline{\mathrm{OE}} \mathrm{E}_{1}$, then $\gamma= \pm \overline{\mathrm{OH}_{1}}$, since $\gamma$ and $\epsilon$ are conjugate. It will thus be seen that $\rho$ has any one of the four values given by

$$
\rho= \pm \mathrm{VOE}_{1} \cdot \overline{\mathrm{OH}}_{1} \text { or } \pm \mathrm{V} \overline{\mathrm{OE}}_{2} \cdot \overline{\mathrm{OH}}_{2} \text {. }
$$

By taking all possible directions for $\rho$, this gives a geometrical construction for the wave-surface. From the construction it is clear that the surface forms a double sheet with centre at 0 . Fresnel's construction is clearly what the present construction degenerates into when one of the ellipsoids is a sphere.

Of course similar remarks apply to the index-surface as depending on the $\mathbf{D}$-oid and B-oid.

Take $\epsilon=\overline{O E}_{1}, \gamma=\overline{O H} H_{1}$. Then by Prop. II. $-\delta^{-1},-\beta^{-1}$ are the perpendiculars from $O$ on the tangent-planes at $\mathrm{E}_{1}$ and $H_{1}$ to the E-oid and $H$-oid. Since $\sigma=V \delta \beta$ the wavefront, supposed drawn through 0 , contains both these normals, 
i. e. it is the plane perpendicular to the line of intersection of the tangent planes. Further, since $\delta=\mathrm{V} \gamma \sigma, \beta=\mathrm{V} \sigma \epsilon$ [Prop.III.] the directions of $\delta$ and $\beta$ are given by saying that they are in the wave-front perpendicular to the projections of $\gamma$ and $\epsilon$ respectively on the wave-front. Thus the directions of all six vectors are given by fig. 5, and the line of intersection of the tangent planes at $\mathrm{E}_{1}$ and $\mathrm{H}_{1}$.

If the two ellipses of fig. 5 are similar and similarly situated, the directions of $\epsilon$ and $\gamma$ are those of any two conjugate diameters of either ellipse. Since the area of the circumscribing parallelogram of either ellipse is constant we get but one value of $\rho(=V \epsilon \gamma)$, including of course its opposite.

The above construction for the wave-front still holds; and we see that the front must, in this case, touch a cone. It is a simple problem, directly from the geometrical construction, to show that this cone is of the second degree. That there is but one pair of directions (and their opposites) of $\rho$ giving this case, is most easily seen by orthogonally projecting the H-oid into a sphere, when we fall on the usual case, as depending on the circular sections of the ellipsoid into which the $\mathbf{E}$-oid has been projected. The geometrical properties connected with conical refraction can thus be treated by the present method. This brief indication of the fact must suffice.

It might be thought that these generalizations of the particular form of theory usually adopted would necessitate a complex proof. On the contrary, it will be found, I think, that the following proofs are simpler than those ordinarily given for the partienlar form.

From Maxwell's 'Theory of Electromagnetism' we take the following; omitting the $4 \pi$ 's, as recommended by Mr. Heaviside, in such work as the present,

$$
\begin{array}{lllll}
\dot{\mathbf{D}}=\mathrm{V} \nabla \mathbf{H}, & \dot{\mathbf{B}}=-\mathrm{V} \nabla \mathbf{E}, & . & . & . \\
\mathbf{D}=c \mathbf{E}, & \mathbf{B}=\boldsymbol{\mu} \mathbf{H}, . \quad . \quad . & . & .
\end{array}
$$

where $c, \mu$ are self-conjugate linear vector-functions of a vector (permittivity and permeability), and

$$
\frac{1}{2}(\mathrm{SDE}+\mathrm{SBH})=-w, \quad . \quad . \quad . \quad .
$$

where $w$ is the energy per unit volume, $i . e$. the "intensity."

The only kind of wave we contemplate is a plane wave in which the quantities have constant value over the wave-front.

Take the axis of $z$ as normal to the front and $k$ as the unit vector in that direction. Denote partial differentiation by $D_{z}$ \&c. Thus if a wave with velocity $v$ can be propagated in 
that direction, we shall have

H $($ or $\mathbf{E} \& c)=$. function $(z-v t)$,

and for any such function

$$
\mathrm{D}_{t}=-v \mathrm{D}_{z}, \quad \nabla=k \mathrm{D}_{\varepsilon}
$$

Thns (1) become;

$$
\mathrm{V} k \mathrm{D}_{z} \mathrm{H}=-v \mathrm{D}_{\dot{z}} \mathrm{D}, \quad \nabla k \mathrm{D}_{z} \mathrm{E}=v \mathrm{D}_{z} \mathbf{B} ;
$$

or putting, in accordance with the definition of $\sigma$ as an indexvector

$$
\begin{gathered}
k v^{-1}=\sigma, \\
\mathrm{D}=-\mathrm{V} \sigma \mathrm{H}, \quad \mathbf{B}=\mathrm{V} \sigma \mathrm{E} .
\end{gathered}
$$

By (4) and (3),

$$
\mathrm{SDE}=\mathrm{S} \sigma \mathrm{EH}=\mathrm{SBH}=-\imath v . \quad . \quad .
$$

By (4) and (5),

$$
\mathrm{VDB} / w=\sigma . . \quad . \quad . \quad \cdot \quad \cdot \quad \cdot
$$

So far we have been practically following Mr. Heaviside.

By (2),

Hence by (5)

$$
\mathbf{S D} d \mathbf{E}=\mathrm{SE} d \mathbf{D}, \quad \mathbf{S B} d \mathbf{H}=\mathbf{S H} d \mathbf{B} .
$$

$$
-\frac{1}{2} d w=\mathrm{SD} d \mathbf{E}=\mathrm{SE} d \mathbf{D}=\mathrm{SB} d \mathbf{H}=\mathrm{SH} d \mathbf{B} . . .
$$

Now define $\rho$ by the equation

By (5),

$$
\begin{aligned}
& \mathrm{VEH} / w=\rho . \\
& \mathrm{S} \sigma \rho=-1 . \\
& \hline
\end{aligned}
$$

By (4),

$$
\begin{aligned}
\mathrm{SH} d \mathrm{~B} & =\mathrm{SH} d \nabla \sigma \mathrm{E}=\mathrm{SH} \sigma d \mathbf{E}+\mathrm{S} d \sigma \mathrm{VEH} \\
& =\mathrm{SD} d \mathrm{E}+w \mathrm{~S} \rho d \sigma .
\end{aligned}
$$

Hence by (7) and (9),

$$
\mathrm{S} \rho d \sigma=0, \quad \mathrm{~S} \sigma d \rho=0 .
$$

This shows that the locus of the extremity of $\rho$ and that of $\sigma$ are polar reciprocals, and therefore that $\rho$ is the vector of ray velocity.

Putting now, in accordance, with our tirst definitions,

$$
\mathrm{D}=\delta \sqrt{ } w, \quad \mathbf{E}=\epsilon \sqrt{ } w, \quad \mathbf{B}=\beta \sqrt{ } w, \quad \mathbf{H}=\gamma \sqrt{ } w, .
$$


and re-writing (4) to (8)

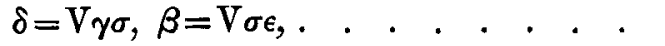

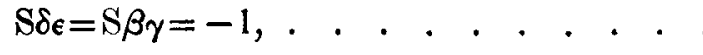

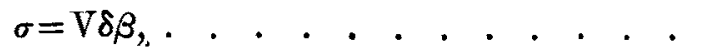

$$
\begin{aligned}
& 0=\mathrm{S} \delta d \epsilon=\mathrm{S} \epsilon d \delta=\mathrm{S} \beta d_{\gamma}=\mathrm{S} \gamma d \beta, \ldots . . . \\
& \rho=V_{\epsilon \gamma} . \text {. . . . . . . . . . }
\end{aligned}
$$

From (2) and (11)

$$
\delta=c \epsilon, \quad \beta=\mu \gamma . \quad . \quad . \quad . \quad . \quad . \quad . \quad . \quad \text {. }
$$

Hence from (13),

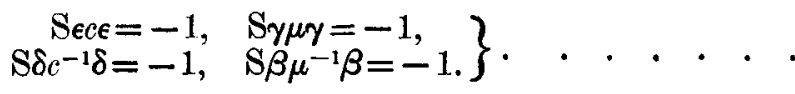

This is Prop. I. Prop. II. is contained in equations (9), $(10),(13),(15)$. Prop. III. is contained in equations (12), (14), (16), and

$$
\epsilon=\mathrm{V} \beta \rho, \quad \gamma=\mathrm{V} \rho \delta, . \quad . \quad . \quad .
$$

which are simple deductions from (16), (12), (13).

To prove Prop. IV. notice that equations (18) are the equations of the E-oid, H-oid, D-oid, and B-oid respectively. From the equations

$$
\mathrm{S} \beta \epsilon=0, \quad \mathrm{~S} \delta \gamma=0
$$

[Prop. III. or eq. (12)] we have by (1i)

$$
\mathrm{S} \epsilon \mu_{\boldsymbol{\gamma}}=0, \quad \mathrm{~S}_{\boldsymbol{\gamma} c \epsilon}=0,
$$

which express that $\epsilon$ and $\gamma$ are conjugate to one another with regard to the $\mathbf{H}$-oid and with regard to the $\mathbf{E}$-oid [these being $S_{\gamma} \mu \gamma=-1$, Sece $=-1$, respectively]. Similarly for $\delta$ and $\beta$.

All these geometrical properties have been proved without finding the equations of the wave-surface and index-surface, and the other well-known pronerties of the wave-surface can be similarly proved. But of course for some purposes it may be desirable to find these equations.

'ine finding of the equation of the index-surface (by essentially the same method as Mr. Heaviside's) may be thus put

$$
\begin{aligned}
c \epsilon=\delta & =V \gamma \sigma=V\left(\mu^{-1} \beta \sigma\right)=-V \sigma \mu^{-1} V \sigma \epsilon \\
& =-m^{-1} \mu \mathrm{V} \mu \sigma \mathrm{V} \sigma \epsilon,
\end{aligned}
$$

where $m$ is the product of the three principal permesbilities. Thus

$$
m \mu^{-1} c \epsilon=\sigma \mathrm{S} \epsilon \mu \sigma-\epsilon \mathrm{S} \sigma \mu \sigma,
$$


therefore

$$
\epsilon / \mathrm{S} \epsilon \mu \sigma=\left(m \mu^{-1} c+\mathrm{S} \sigma \mu \sigma\right)^{-1} \sigma
$$

whence, operating by $\mathbf{S} \mu \sigma()$,

$$
1=\mathrm{S} \sigma \mu\left(m \mu^{-1} c+\mathrm{S} \sigma \mu \sigma\right)^{-1} \sigma,
$$

and this may be put in a variety of forms, as usual. But the interesting thing to notice is that by the above method it is obvious that by a similar process the equation of the wavesurface is

$$
1=\operatorname{S} \rho \mu^{-1}\left(m^{-1} \mu c^{-1}+\operatorname{S} \rho \mu^{-1} \rho\right)^{-1} \rho,
$$

and that this has been arrived at without any complex integration of (9) and (10).

In concluding this part of the paper I may remark that the above methods can be applied to other theories of light. They are not quite so naturally applicable because in such theories-without exception as far as I know-only one of the pairs of vectors $\delta, \epsilon$ and $\beta, \gamma$ obviously presents itself, and the other pair must be deliberately introduced by definition. In the electromagnetic theory both pairs are present before the special problems connected with optics are considered.

\section{Reflexion and Refraction at the Surface of Crystals, treated by a Theorem of Sir Wm. Rowan Hamilton.}

In his 'Elements of Quaternions' (423 (12) in 1st edition, probably this important theorem will not escape the editor of the 2nd edition) Hamilton has proved a theorem of such singular beauty and ease of application in particular cases, that $I$ think it well worth calling attention to. This seems the more necessary as it appears to have been overlooked by subsequent writers.

His treatment is - probably necessarily, based as it is on MacCullagh's theory - somewhat complex, whereas the treatment on the electromagnetic theory is little more than an interpretation of the fundamental equations. He treats only of the case where a ray in an isotropic medium is incident on the face of a crystal, and where the polarization of the incident light is such that there is but one refracted ray. On the electromagnetic theory it is just as easy to suppose both media to be crystalline, and to allow the incident polarization to be arbitrary.

There will in this case be one incident ray, two refracted rays, and two reflected rays, five in all as against Hamilton's (and MacCullagh's, since in this particular Hamilton is following MacCullagh) three.

Let the M.M.F.'s of the five rays at the point of incidence 
(taken as the point called $O$ above) at any instant be, in order,

$$
\mathbf{H}, \mathbf{H}_{1}{ }^{\prime}, \mathbf{H}_{2}{ }^{\prime}, \mathbf{H}_{1}{ }^{\prime \prime}, \mathbf{H}_{2}{ }^{\prime \prime} \text {, }
$$

and the corresponding vectors of ray-velocity

$$
\rho, \rho_{1}{ }^{\prime}, \rho_{2}{ }^{\prime}, \rho_{1}^{\prime \prime}, \rho_{2}^{\prime \prime} \text {, }
$$

and similarly for the other vectors involved.

The theorem is true only for the usual form of electromagnetic theory, i.e. for the case when both media are assumed non-magnetic. It is to be remembered that this form is apparently as satisfactory as the more general form in explaining all known optical phenomena. The following is the theorem :-

If we suppose mechanical forces

$$
\mathbf{H},-\mathbf{H}_{\mathbf{1}}{ }^{\prime},-\mathbf{H}_{\mathbf{2}}{ }^{\prime}, \mathbf{H}_{1}{ }^{\prime \prime}, \mathbf{H}_{\mathbf{2}}{ }^{\prime \prime}
$$

to act at points whose vector coordinates are

$$
\rho, \rho_{1}{ }^{\prime}, \rho_{2}{ }^{\prime}, \rho_{1}{ }^{\prime \prime}, \rho_{2}{ }^{\prime \prime},
$$

they will reduce to a couple whose plane is parallel to the face. [See figures 6 and 7 below.]

Before giving the proof some remarks are desirable. If the incident wave-front and the two wave-surfuces (centre at point of incidence) be given, by Huyghens's construction we know the five wave-fronts. These fronts, supposed drawn touching the wave-surfaces at the appropriate points, all contain the line of intersection of the incident front with the face, or as we shall call it the trace of that front on the face. Since the fronts are known the directions of the M.M.F'.'s are known, and it only remains to find their magnitudes. These M.M.F.'s, drawn as described in the enunciation, are in the corresponding fronts, and therefore all intersect one line, the trace.

Thus when the circumstances of incidence are given the four magnitades of the M.M.F.'s of the refracted and reflected waves are required. The theorem gives five conditions, but one of them is always satisfied by reason of the five vectors all intersecting one line. The theorem is in every case not only necessary, but sufficient to determine the unknown quantities.

In the proof about to be given, whenever $\Sigma \pm()$ is applied to a sum, referring to the five rays, the sign + is to be understood to refer to one medium, and the sign - to the other.

Let $k$ be the unit normal of the face. The boundary conditions are that the tangential components of $\mathbf{H}$ and $\mathbf{E}$, and 
the normal components of $\mathbf{B}$ and $\mathbf{D}$, are continuous. Thus [Prop. III.],

$$
0=\Sigma \pm V k \mathbf{H}, \quad 0=\Sigma \pm V k \mathbf{E}=\Sigma \pm V k V B \rho
$$

$$
0=\Sigma \pm \mathbf{S} k \mathbf{B}, \quad 0=\Sigma \pm \mathbf{S} k \mathbf{D} .
$$

[Here we have apparently six conditions to determine four unknowns. That the two last conditions are contained in the others may be thus shown:- $\sigma, \sigma_{1}^{\prime} \ldots$ are perpendicular to the fronts and therefore to the trace. Their components parallel to the face are equal ; for expressing that the foot of the perpendicular from 0 on the trace is in each front

$$
-1=\mathrm{S} \alpha \sigma=\mathrm{S} \alpha \sigma_{1}^{\prime}=\ldots,
$$

where $\alpha$ is the vector perpendicular from 0 on the trace. Hence

$$
\mathrm{V} k \sigma=\mathrm{V} k \sigma_{1}^{\prime}=\ldots=\eta,
$$

say, where $\eta$ is parallel to the trace. But

$$
\Sigma \pm S k B=\Sigma \pm S k \sigma E=\Sigma \pm S \eta E=0
$$

by the condition $\Sigma \pm V k E=0$. Similarly the condition $\Sigma \pm S k D=0$ can be deduced from $\Sigma \pm V k H=0$. We shall, however, use the condition $\Sigma \pm S k B=\overline{0}$.]

Make now the assumption that the media are non-magnetic, i.e. that $\mathbf{B}$ is parallel and proportional to $\mathbf{H}$. In this case both normal $(\Sigma \pm S k B=0)$ and tangential $(\Sigma \pm V k \mathbf{H}=0)$ components of $\mathrm{H}$ are continuous, $i$. $e$.

$$
\Sigma \pm \mathbf{H}=0 \text {. }
$$

Also from the condition $\Sigma \pm V k E=0$,

$$
\Sigma \pm V k V \rho \mathbf{H}=0 \text {. }
$$

The first of these expresses that the mechanical system of the enunciation reduces to a couple. The second expresses that the vector moment of the forces about $O$ is parallel to the normal of the face, i.e. that the plane of the couple is parallel to the face. This is Hamilton's theorem.

It will be noticed also that if the conditions of the enunciation are satisfied, the boundary conditions are satisfied. Thus the theorem is always sufficient to give the four unknowns.

To exhibit the ease of application in particular cases to useful interpretations take the case of two isotropic media. The theorem, it will be noticed, was not arrived at in considering this comparatively simple case, but it seems to me to serve as a much better resumé of our knowledge, even in this case, than any other I have come across. 
First, let $\mathrm{H}$ be in the plane of incidence, i.e. let the incident light be polarized in the plane of incidence. Fig. 6 indicates

Fig. 6.

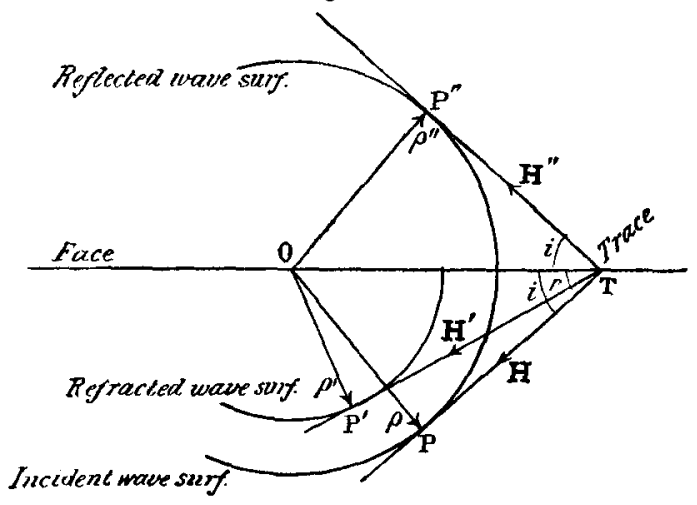

the nature of the reflected and refracted light. The plane of incidence is the plane of the paper, $i$ the angle of incidence, and $r$ the angle of refraction; $\rho, \rho^{\prime}, \rho^{\prime \prime}$ are the vectors of rayvelocity of the incident, refracted, and reflected light. $\mathbf{H}$ is drawn in the incident wave-front and in the plane of incidence. It will be seen that if $\mathbf{H}^{\prime}$ and $\mathbf{H}^{\prime \prime}$ be also drawn in the refracted and reflected fronts and in the plane of incidence, as indicated in the figure, three mechanical forces $\mathbf{H},-\mathbf{H}^{\prime}, \mathbf{H}^{\prime \prime}$ may be made to be in equilibrium, i.e. to reduce to a (null) couple whose plane is parallel to the face. By the sine rule for the equilibrium of three concurrent forces, we have

$$
\frac{\mathrm{TH}}{\sin (i+r)}=\frac{\mathrm{T} \mathbf{H}^{\prime}}{\sin 2 i}=\frac{\mathrm{TH}^{\prime \prime}}{\sin (i-r)^{\prime}}
$$

in which it is to be remarked [eq. (5) above] that the intensities are proportional to the squares of the numerators and therefore to the squares of the denominators.

Fig. $\dot{b}$ also serves for the case when the light is polarized perpendicular to the plane of incidence. $\mathbf{H}, \mathbf{H}^{\prime}$, and $\mathbf{H}^{\prime \prime}$ are not then as indicated in the figure, but instead they act at the points $\mathrm{P}, \mathrm{P}^{\prime}$, and $\mathrm{P}^{\prime \prime}$ of the wave-surfaces perpendicular to the plane of the paper. In order that in this case the system may reduce to a couple parallel to the face, $\mathbf{H}$ and $\mathbf{H}^{\prime \prime}$ must have a resultant equal and parallel to $\mathbf{H}^{\prime}$ at the same distance as $\mathbf{H}^{\prime}$ from the face and on the same side of the face. Since the distances of $\mathrm{P}, \mathrm{P}^{\prime}$, and $\mathrm{P}^{\prime \prime}$ from the face are

OT $\cos i \sin i, \mathrm{OT} \cos r \sin r$, and OT $\cos i \sin i$ 
respectively, these conditions give

or

$$
\frac{\mathrm{TH}}{\sin 2 i+\sin 2 r}=\frac{\mathrm{T}^{\prime} \mathrm{TH}^{\prime \prime}}{\sin 2 i-\sin 2 r}=\frac{\mathrm{TH}^{\prime}}{2 \sin 2 i},
$$

$$
\frac{\mathrm{TH}}{\sin (i+r) \cos (i-r)}=\frac{\mathrm{TH}^{\prime}}{\sin 2 i}=\frac{+\mathrm{TH}^{\prime \prime}}{\cos (i+r) \sin (i-r)},
$$

which give the ratios of the intensities in this case.

Lastly, take the case of two crystalline media. Denote in this case the angles of incidence, refraction, and reflexion by

$$
\psi, \psi_{1}^{\prime}, \psi_{2}^{\prime},-\psi_{2}^{\prime \prime},-\psi_{2}^{\prime \prime} \text {. }
$$

[The angles of reflexion are called $-\psi_{2}^{\prime \prime},-\psi_{2}^{\prime \prime}$ because the corresponding fronts are on the side of the face opposite to that on which are the other fronts.]

In fig. 7 let $O P$ be the incident ray, $O S$ the perpendicular from 0 on the front, OT the perpendicular on the trace, $\mathrm{K}$ the

Fig. 7.

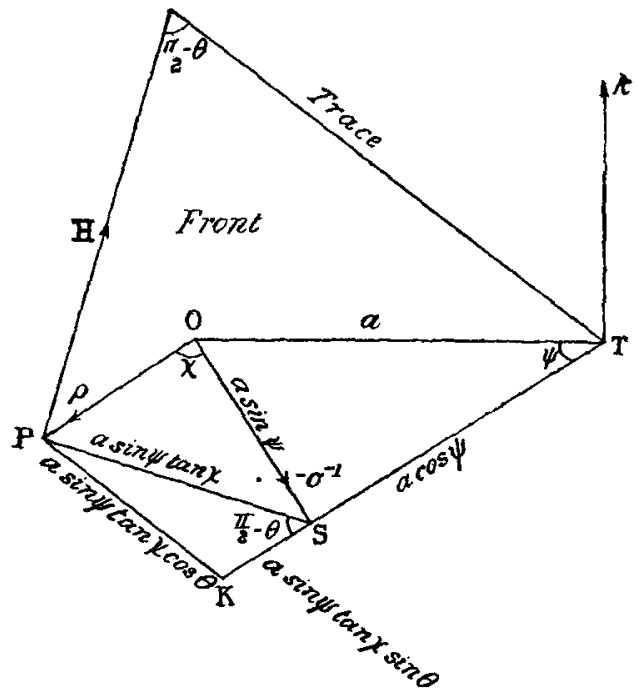

point of intersection of TS and the parallel through $\mathrm{P}$ to the trace, $\chi$ the angle POS between the ray and the wave normal, $\frac{1}{2} \pi-\theta$ the angle between $\mathbb{H}$ and the trace, and $a$ the length of OT. Since $H$ is perpendicular to $\rho$, and therefore to PS, the angle PSK $=\frac{1}{2} \pi-\theta$. The lengths, in terms of $a$ and the angles, of the various lines, are sufficiently obvious from the figure.

Putting $\mathrm{H}$ for $\pm \mathrm{TH}$, the components of $\mathrm{H}$ parallel to the Phil. Mag. S. 5. Vol. 49. No. 297. Feb. 1900. 
normal of the face, to $\mathrm{OT}$, and to the trace are respectively

$\mathrm{H} \cos \theta \sin \psi, \mathrm{H} \cos \theta \cos \psi, \mathrm{H} \sin \theta$.

Hence, using the theorem by resolving in these directions and taking moments round $\mathrm{OT}^{\mathrm{T}}$,

$0=\Sigma \pm H \cos \theta \sin \psi=\Sigma \pm H \cos \theta \cos \psi=\Sigma \pm H \sin \theta$, and

$0=\Sigma \pm H\left\{\sin ^{2} \psi \tan \chi \cos ^{2} \theta+\sin \psi \sin \theta(\cos \psi+\sin \psi \tan \chi \sin \theta)\right\}$

O)

$0=\Sigma \pm H \sin \psi(\sin \psi \tan \chi+\sin \theta \cos \psi)$.

These reduce to equations (49) $\S 420$ of Basset's 'Physical Optics' when the incident medium is isotropic.

I may remark that I believe this theorem of Hamilton's is nowliere referred to in the treatise just mentioned, nor in Lord Rayleigh's article "Wave Theory," in the Encyclopedia Britannica, 9th ed., nor in Glitzebrook's 'Report on Optical 'Theories,' in the B A. Reports, 1885.

University of Tasmania, Hobart, 20 th November, 1899.

XIX. Intelligence and Miscellaneous Articles.

CHEMICAL EHFHCTS PRODUCED BY BECQUEREL'S RAYS.

BY M. P. AND MME. CURIE.

THE rays emitted by very active radiferous salts of barium are capable of converting oxygen into ozone. When the radioactive salt is kept in a stoppered bottle, a very distinct odour of ozone is perceived on opening the bottle. M. Demarçay discovered this pbenomenon with some very active radiferous barium chloride which we had sent him, for his spectroscopic researches, in a little stoppered bottle. When the flask is opened the odour is incompletely dissipated ; in order that it may regain its original strength it is sufficient to close the flask some ten minutes.

We bave verified the production of ozone by starched potassiumiodide paper, which becomes lightly tinted when placed before the mouth of the bottle. The tint is deeper when the radiferous barium chloride is brought in contact with the paper, whilst ordinary barium chloride under the same conditions produces no effect.

The radiferous compounds necessary for the production of onone are all very active and all luminous. The phenomenon seems more nearly related to the radioactivity than to the luminosity. Thus a very luminous carbonate of radium produces less ozone than a chloride of radium less luminous but more strongly radioactive.

We have likewise remarked a colouring action of Becquerel's rays on glass. If a radium salt is kept for some time in a glass flask, a violet coloration is perceived which appears to spread itself from the interior of the flask towards the exterior. With a 